See discussions, stats, and author profiles for this publication at: https://www.researchgate.net/publication/308699625

\title{
The Influence of a Personal Trainer on Self-Selected Loading during Resistance Exercise
}

Article in The Journal of Strength and Conditioning Research · September 2017

DOI: $10.1519 / \mathrm{JSC} .0000000000001663$

CITATIONS

2

4 authors, including:

Marcelo Ricardo Dias

Instituto Metodista Granbery - Colégio e Faculdade

42 PUBLICATIONS 84 CITATIONS

SEE PROFILE

Nicholas Ratamess

The College of New Jersey

267 PUBLICATIONS 11,918 CITATIONS

SEE PROFILE
371

Francisco Jose Félix Saavedra

Universidade de Trás-os-Montes e Alto Douro

77 PUBLICATIONS 122 CITATIONS

SEE PROFILE

Some of the authors of this publication are also working on these related projects:

Project Validação de Instrumentos de Avaliação Psicológica no Contexto Desportivo View project

Project Self-selected and imposed intensity in aerobic and resistance training View project 


\title{
The Influence of a Personal Trainer on Self-Selected Loading during Resistance Exercise
}

\author{
Marcelo R C Dias ${ }^{1}$, Roberto Simao ${ }^{2}$, Francisco J F Saavedra ${ }^{3}$, \\ and Nicholas A Ratamess ${ }^{4}$
}

Running Head: Self-Selected Loads and Personal Trainer

\section{${ }^{1}$ Marcelo R C Dias [Corresponding Author]}

Affiliations: Laboratory of Exercise Physiology and Morphofunctional Assessment, Granbery Methodist College, Juiz de Fora, Brazil. Post Graduate Program in Sport Science, University of Trás-os-Montes and Alto Douro (UTAD), Vila Real, Portugal.

Addresses: Rua Floriano Peixoto, 937 / 503 - Centro, Juiz de Fora, MG, 36.015-440, Brazil. Telephone: 0055(32)991940154. E-mail: diasmr@gmail.com

\section{${ }^{2}$ Roberto Simão}

Affiliations: School of Physical Education and Sports, Rio de Janeiro Federal University, Rio de Janeiro, Brazil.E-mail: rsimaoj@terra.com.br

\section{${ }^{3}$ Francisco J F Saavedra}

Affiliations: Post Graduate Program in Sport Science, University of Trás-os-Montes and Alto Douro (UTAD), Vila Real, Portugal. Research Center for Sport, Health, and Human Development, University of Trás-os-Montes and Alto Douro (UTAD), Vila Real, Portugal. E-mail: fjfsaave@utad.pt

\section{${ }^{4}$ Nicholas A Ratamess}

Affiliations: Department of Health and Exercise Science, The College of New Jersey, Ewing, NJ 08628: E-mail: ratamess@ @tcnj.edu 


\section{ABSTRACT}

2 The purpose of this study was to compare differences in muscle strength and self-selected resistance training intensities between trained subjects who trained under the supervision of a

4

5 personal trainer (PT) and those who trained without supervision (WoPT). Twenty-one trained subjects, men $(n=12)$ and women $(n=9)$, completed three sessions (separated by 48 hours) in the following sequence: $\left(1^{\text {st }}\right)$ self-selected intensity assessment consisting of performance of three sets of 10 repetitions for the leg press, bench press, leg extension, and arm curl exercises with self-selected load; $\left(2^{\text {nd }}\right)$ a one repetition-maximum (1RM) test to determine subjects' maximal strength in the four exercises; and $\left(3^{\text {th }}\right)$ a 10 repetition-maximum $(10 \mathrm{RM})$ test to determine the maximum load completed for 10 repetitions for each exercise. Selfselected training loads were significantly higher in PT compared to WoPT for the leg press (by 15.6\%), bench press (by 26.6\%), leg extension (by 12.1\%), and arm curl (by 22.2\%) exercises. Self-selected training loads expressed relative to $1 \mathrm{RM}$ and $10 \mathrm{RM}$ data were significantly higher in PT (49\% to $59.5 \%$ of $1 \mathrm{RM} ; 62.7 \%$ to $77.3 \%$ of $10 \mathrm{RM}$ ) than WoPT ( $41 \%$ to $58.7 \%$ of $1 \mathrm{RM} ; 58.7 \%$ to $76.2 \%$ of $10 \mathrm{RM}$ ) with largest difference observed in the lower-body exercises. Ratings of perceived exertion (RPE) values were significantly higher in PT compared to WoPT. The results of the present study indicated that supervised resistance training with a personal trainer was advantageous in trained subjects although selfselected loading was still considerably lower than 1RM and 10RM percentage values.

Keywords: Resistance Training, Strength Training, Supervision, Ratings of Perceived Exertion 


\section{INTRODUCTION}

The ability to generate high levels of muscle force is a key health-related fitness component. Enhanced muscle strength is an important quality for athletic success and for performance of several activities of daily living. Therefore, increasing muscle strength through progressive overload has many advantages and muscular strength improvements are the focus of many resistance training programs (9). Adaptations to resistance training enable greater force generation through numerous mechanisms, both morphological and neuromuscular. However, studies show that the strength-enhancing effect is only brought about when a threshold intensity is consistently targeted in training $(9,12)$. Often, the selfselected training intensity may fall below the individual's threshold intensity needed for strength improvements (10) and may be lower than general recommendations from professional health organizations (12). For example, Glass and Stanton (5) reported that women self-selected intensities ranging from $\sim 40$ to $52 \%$ of their $1 \mathrm{RM}$ and ratings of perceived exertion (RPE) across all exercises assessed which was significantly lower than recommended values for the repetition range investigated (9). Focht (3) reported that women self-selected a resistance that was on average $56 \%$ of their 1RM and low RPE during performance of the leg extension, chest press, pull-down, and overhead press exercises. Collectively, these studies demonstrate a propensity for individuals to target loads and RPEs during resistance exercise that may be suboptimal based on established strength training guidelines.

Personal trainers provide valuable services to their clients. They design resistance training programs (i.e., workout structure, exercise selection and sequence, frequency, intensity, volume, lifting velocity, and rest intervals) based on established guidelines and recommendations (9), instruct and correct exercise techniques, motivate their clients, and provide psychological reinforcement in progression towards goal attainment (10). The 
encouragement to train at a higher relative intensity and the direct supervision by a personal trainer may expose clients to a more favorable training stimulus $(1,10)$. However, supervised training under the guidance of a personal trainer may be expensive and a resource that is not always available at certain facilities. It has been estimated that approximately $13 \%$ of trainees utilize personal trainers (7). Thus, the benefits must be weighed against the costs when deciding upon the use of personal trainers.

Conventional wisdom suggests that training with a personal trainer may be more beneficial for improving health- and skill-related fitness components than training without supervision $(2-5,8,10)$. Mazzetti et al. (8) first reported that leg press and bench press rate of strength gains were significantly higher in a supervised training group compared to a nonsupervised training group. Subsequently, other studies have confirmed benefits of supervised training $(2,10,14)$. Ratamess et al. (10) reported the average self-selected intensity for all exercises (chest press, leg press, seated row, and leg extension) was $\sim 51.4 \%$ of $1 \mathrm{RM}$ in a group supervised group by personal trainers versus $\sim 42.3 \%$ of $1 \mathrm{RM}$ in a non-supervised group. Other studies reported supervised training groups have greater adherence to training (2) and higher RPE during resistance exercise (10).

Currently, few published studies $(8,10,14)$ have examined the benefits of supervised personal training compared to non-supervised training programs, especially in trained subjects. For example, Focht (3) showed that the self-selected load, ie, submaximal, differs from imposed load which is next of maximal. Because of the paucity of existing data and differences in study design, it is difficult to draw firm conclusions regarding the influence of supervision of resistance training on resistance exercise performance. Therefore, the purpose of the present study was to compare differences in muscle strength and self-selected resistance training load between trained subjects who trained under the supervision of personal trainers compared to those subjects who trained unsupervised. A secondary purpose 
was to compare the relative training intensities of both groups to recognized guidelines from major strength training and conditioning organizations (9). We hypothesized that trained subjects training under the supervision of a personal trainer would self-select greater loads and have significantly greater muscle strength than those trained on their own.

\section{METHODS}

\section{Experimental Approach to the Problem}

In order to acutely compare differences in muscle strength and self-selected resistance training loads between trained subjects who trained with a personal trainer or without a personal trainer, subjects completed three sessions (separated by 48 hours) in the following sequence: $\left(1^{\text {st }}\right)$ self-selected intensity assessment consisting of performance of three sets of 10 repetitions for the leg press, bench press, leg extension, and arm curl exercises with selfselected load; $\left(2^{\text {nd }}\right)$ a one repetition-maximum $(1 \mathrm{RM})$ test/re-test to determine subject's maximal strength in the four exercises; and $\left(3^{\text {nd }}\right)$ a 10 repetition-maximum $(10 \mathrm{RM})$ test/retest to determine the maximum load completed for 10 repetitions for each exercise. Selfselected protocol loads, 1RM and 10RM strength data, and RPEs were recorded for each exercise. This acute study design allowed us to precisely compare performance characteristics of subjects who consistently trained under direct supervision versus subjects who trained on their own. We hypothesized that muscle strength and self-selected loads would be higher in the group who consistently trained under direct supervision.

\section{Subjects}

Twenty-one resistance-trained men $(n=12)$ and women $(n=9)$ with at least 12 months of experience volunteered to participate in this study. Subjects were subsequently assigned to either the personal trainer - PT ( $n=8 ; 6$ men and 2 women) or without personal 
1 trainer - WoPT ( $n=13 ; 9$ men and 4 women) group. Because Glass and Stanton (5) did not

2 observe differences in load selection between genders, we decided to pool men and women

subjects into heterogeneous groups. All subjects reported strength and hypertrophy gains as their major goals of resistance training. The PT group was comprised of subjects who were currently training under the supervision of a personal trainer (i.e., for at least two days per week for six months). Subjects in the WoPT group trained on their own for at least six months prior to initiating the study. Potential subjects were randomly recruited from training facilities at the university via advertisements and through "word of mouth". Group subject characteristics are presented in Table 1. No significant differences in subject characteristics (age, body mass, height, BMI, body fat and RT frequency) was observed between groups. In addition, subjects had no medical or orthopedic problems that compromised their participation or performance in this study. Subjects read and signed an informed consent document that had been approved by the university's ethics committee in conformity with the Helsinki Declaration.

*** Insert Table 1 here ***

\section{Self-Selected Loading Assessment}

After a general warm-up, each subject was carefully instructed to select a resistance they would typically use in their own workouts for completion of 10 repetitions (or until they reached failure) during the initial testing session. Subjects were given multiple opportunities to select the appropriate weight (i.e., if the initial selection appeared to be too light or heavy) and the investigator provided no additional information that could have created bias in the weight selection. The exercises selected for assessment were the $45^{\circ}$ leg press (LP), bench press (BP), leg extension (LE), and EZ bar arm curl (AC). The exercises were performed in the order listed. The LP and LE exercises were performed using Righetto® resistance training 
machines (High On, São Paulo, Brazil) and BP and AC exercises were performed using free weight. Each subject completed three sets of each resistance exercise at their self-selected load using a 90-second rest interval in between sets and exercises. The training load was assessed following completion of each set. Testing was conducted without the presence of personal trainer for the PT group to avoid any potential influence the trainer could have on load selection.

\section{Strength Testing}

Following completion (48 hours) of self-selected load testing, subjects were assessed for their $1 \mathrm{RM}$ and 10RM maximal strength using previously validated procedures (12). All exercises were tested on the same day in the same order performed in the self-selected training intensity session. The 1RM and 10RM tests were conducted in a randomized and counterbalanced order on nonconsecutive days (see Figure 1). Forty-eight hours after each test (1RM and 10RM), a retest was performed to determine reliability. The highest load achieved on any test day was considered to be the 1RM and 10RM load, respectively, for each exercise. Subjects were not allowed to exercise in between testing sessions. All 1RM and 10RM values were determined within five sets to avoid excessive fatigue. Rest intervals between sets were four min and 10 min between the different exercises (12).

To minimize error during testing, all subjects received standard instructions concerning correct exercise technique; all testing sessions were strictly supervised by research staff, and all subjects received the same verbal encouragement each sets for all subjects. In addition, all subjects performed a standard warm-up consisting of three sets each of the first two exercises (LP and BP) for 10, 5, and 3 repetitions with progressive loading, respectively. The machine settings for strength testing were identical to those used in the selfselected resistance exercise protocol. 
Measures

Session training load was determined as weight lifted in $\mathrm{kg}$ for a specific exercise.

Intensity was calculated as the average percentage of 1RM. Ratings of perceived exertion (RPE) were obtained following each set (RPE muscle) of resistance exercise and at the end of the training (RPE overall) utilizing the 10-point OMNI-RES scale (11). Subjects were provided with explicit written and verbal instructions to accurately gauge their level of effort.

Data reported are the means $( \pm \mathrm{SD})$ for each exercise and the RPE values for the entire protocol.

\section{Statistical Analyses}

Descriptive statistics (means $\pm S D$ ) were calculated for all dependent variables. Statistical power was calculated for each variable and was $>0.80$. Shapiro-Wilk and Levene's tests were used to check normality and homogeneity between groups. An independent t-test was performed to detect differences between groups. A 2 (group) x 4 (exercise) analysis of variance (ANOVA) was used to examine differences in strength performance and RPE. When a significant difference was shown via ANOVA, a Tukey post hoc analysis was performed to determine where significant differences existed between means. Cohen's effect size was calculated to determine the magnitude of differences in the self-selected loads, 1RM, and 10RM data. For all analyses the 0.05 level of significance was used.

\section{RESULTS}

Excellent day-to-day 1RM and 10RM reliability for each exercise was shown using the study protocol. The 1RM for the two testing sessions separated by 48 hours showed 
1 interclass correlation coefficients of: LP, $r=0.96$; BP, $r=0.99$; LE, $r=0.97$; and AC, $r=$

2 0.99. The 10RM tests showed interclass correlation coefficients of: LP, $r=0.98$; BP, $r=$

0.99; LE, $r=0.97$; and AC, $r=0.99$. Additionally, paired Student's t-tests showed no significant difference between the two testing sessions for the 1RM or 10RM test for any exercise tested.

Self-selected loads for each exercise are presented in Table 2. Mean self-selected loads per exercise were significantly higher in PT compared to the WoPT group. Selfselected loads in PT group were 12.1 to $26.6 \%$ higher than those selected by the WoPT group. The effect size was small to medium for the difference in self-selected loads between the PT and WoPT groups.

$$
\text { *** Insert Table } 2 \text { near here *** }
$$

The 1RM and 10RM data, as well as the relative percent of the self-selected loads, are presented in Figure 2. One repetition-maximum and 10RM values were significantly higher in PT for three of the four exercises tested compared to WoPT. Significant differences were observed between groups in the self-selected load percentage of 1RM and 10RM. The relative 1RM percentages were significantly higher in the PT group for three of the four exercises with the exception of the bench press, i.e., presented medium to large effect sizes in the lower-body exercises and small effect sizes in the upper-body exercises. The relative 10RM percentages presented medium effect sizes for two of the four exercises but only a small to medium effect.

$$
\text { *** Insert Figure } 2 \text { here *** }
$$

The RPE presented small differences, i.e., effect size $<0.3$ in all exercises, which RPE values for each exercise were significantly higher $(P<0.05)$ in PT group compared to 
the WoPT group: leg press $=7.92 \pm 1.4(\mathrm{PT})$ versus $7.21 \pm 1.0(\mathrm{WoPT})$; bench press $=7.29 \pm$ $2.6(\mathrm{PT})$ versus $6.85 \pm 1.9(\mathrm{WoPT})$; leg extension $=7.79 \pm 1.4(\mathrm{PT})$ versus $6.97 \pm 1.5$ $(\mathrm{WoPT})$; and arm curl $=7.88 \pm 1.2(\mathrm{PT})$ versus $7.36 \pm 1.5(\mathrm{WoPT})$. The RPE overall was also significantly higher $(P<0.05)$ in PT $(7.75 \pm 0.7)$ compared to WoPT $(7.23 \pm 1.0)$.

\section{DISCUSSION}

A critical finding from the present investigation was that trained subjects who trained under the supervision of a personal trainer self-selected significantly greater loads during the leg press, bench press, leg extension, and arm curl exercises compared to those subjects who train on their own. However, self-selected intensity of resistance exercise was considered to be relatively low in both groups especially for lower-body exercises. RPE was low for both groups. The self-selected intensities by both groups were, on average, lower than the recommended values for resistance training progression when performing 10-repetition sets (10).

The results of the present study support previous research demonstrating superiority of supervised resistance training. Mazzetti et al. (8) reported that 12 weeks of supervised resistance training promoted greater increases in 1RM squat and bench press compared to strength increases seen in an unsupervised group. Ratamess et al. (10) investigated women who trained under the supervision of a personal trainer versus those who did not and reported that women who trained with a personal trainer self-selected intensities in a range of $43 \%$ to $57 \%$ of $1 \mathrm{RM}$ for all exercises (chest press, leg press, seated row, and leg extension), or an average of $51.4 \%$ of $1 \mathrm{RM}$ versus an average of $42.3 \%$ of $1 \mathrm{RM}$ in the unsupervised group. Recently, Storer et al. (14) reported chest press and leg press strength gains of $42 \%$ and $35 \%$ versus $19 \%$ and $23 \%$, respectively, in a personally trained group versus an unsupervised group. In addition, only the PT group significantly increased lean body mass and peak leg 
1 power (14). Therefore, these results support previous research showing greater maximal

strength increases through supervised resistance training and demonstrate a benefit to use of personal trainers $(8,10)$.

The self-selected intensity by subjects in the PT group ranged from $47.8 \%$ to $61.5 \%$ of 1RM whereas the WoPT group ranged from $42.1 \%$ to $61.2 \%$ of $1 \mathrm{RM}$ for the four exercises assessed. The average self-selected load for all exercises was $54.0 \%$ of $1 \mathrm{RM}$ in the PT group and $49.8 \%$ of $1 \mathrm{RM}$ in the WoPT group. These intensities may be considered typical for general fitness resistance training but fall below recommended values needed for strength training progression (9). Other studies have shown subjects tend to self-selected low intensities for strength development, i.e., 40 to $56 \%$ of their $1 \mathrm{RM}(3,5)$. These values $(<60 \%)$ can be effective for untrained subjects that benefit from strength and hypertrophy gains (13).

Interestingly, the relative percentage of self-selected loads for upper-body exercises (bench press and arm curl) was higher than those self-selected loads for the lower-body exercises (leg press and leg extension) independent of training group in the present study. These results confirm the findings of Ratamess et al. (10) in resistance-trained women who also reported lower self-selected loads for the leg press and leg extension exercises compared to the chest press and seated row. This discrepancy likely occurred because upon a posttesting interview, Ratamess et al. (10) reported that women appeared to have a general concern about gaining excessive muscle mass in the lower body. However, the concern was not as prevalent in women who trained under the supervision of a personal trainer indicating that education from the trainers helped to dispel the myth of excessive hypertrophy in the lower body (10). This may help explain the self-selection of lighter loads for lower-body exercises (7). In contrast, the relative percent selected in the Glass and Stanton (5) study in men and women for the chest, back, and shoulder exercises were slightly lower than that 
1 observed for the leg press. Nevertheless, our data support those studies $(7,10)$ demonstrating

2 lower relative load selection for lower-body exercises. The reason for the contrast may be related to capacity of men and women to sustain the load, although Glass and Stanton (5)

4 have not found differences between genders. In addition, the subjects in the Ratamess et al. study (10) also reported being surprised with the magnitude of their 1RMs for the lower-body exercises. Our data confirm these findings as many of the subjects in the present study reported post testing astonishment by the amount of weight they were able to lift during the $1 \mathrm{RM}$ and 10RM tests. Most of these subjects had not previously trained at a relative intensity close to these values. Thus, their relative self-selected loads may have been underestimated due to possessing greater strength than anticipated.

Regardless of personal training status, all subjects in the present study self-selected loads that could be considered below a relative intensity needed for progression during strength training (9). Although increasing muscle strength is only one of several goals associated with resistance training, our data and the results of other studies $(3,5,10)$ indicate that there is a tendency in health clubs for subjects to select lighter weights given the targeted repetition scheme (i.e., 10 repetitions). These self-selected intensities fell below 67\% of 1RM. In the PT group, $37.5 \%$ of subjects self-selected a intensity of at least $67 \%$ of $1 \mathrm{RM}$ in one exercise, $12.5 \%$ self-selected intensity of at least $67 \%$ of $1 \mathrm{RM}$ in two exercises, and no subject self-selected a intensity of at least $67 \%$ of $1 \mathrm{RM}$ in three or four of the exercises. Only one subject self-selected an intensity of at least $80 \%$ of $1 \mathrm{RM}$ in more than one exercise. In the WoPT group, $38.5 \%$ of subjects self-selected a intensity of at least $67 \%$ in at least one of the exercises and $7.7 \%$ of subjects self-selected a intensity of at least $67 \%$ of 1RM in two exercises. Interestingly, $37.5 \%$ and $76.9 \%$ of subjects in the PT and WoPT groups, respectively, self-selected weights that were less than $50 \%$ of $1 \mathrm{RM}$ in more than two 
exercises. These data indicate that several subjects self-selected loads that are considered light-to-moderate for 10-repetiton sets.

Subjects in the PT group reported higher RPE values for each exercise compared to the WoPT group. RPE values in the PT group were $6 \%$ to $10.5 \%$ higher for all 4 exercises compared to the WoPT group. Overall, the mean RPE in the PT group was $6.7 \%$ higher than the WoPT group. These data reflect the heavier loading selected by the PT group and indicate that trained subjects who train with a personal trainer are accustomed to training at a higher level. These data confirm results from Ratamess et al. (10) who reported that subjects who trained with a personal trainer reported higher RPE values for three of four exercises tested. Because personal trainers prescribed the intensity to the subjects in the PT group, it is likely that these subjects were accustomed to higher levels of exertion in their workouts than the WoPT group. This beneficial effect appeared to carryover to the protocol used in the present study when subjects were tested in the absence of their personal trainers.

In summary, the results of the present study indicated that trained subjects who trained under the supervision of a personal trainer self-selected significantly greater loads during the leg press, bench press, leg extension, and arm curl exercises compared to those subjects who train on their own. The importance of a personal trainer was noted as they prescribe exercises and educate clients on several concepts of health and fitness. These results support previous studies demonstrating the superiority of supervised resistance training $(8,14)$. Of significance was the finding that both groups self-selected loads that fell below recommended values for strength training progression (9).

\section{PRACTICAL APPLICATIONS}

Overload is a critical component of resistance training that leads to gains in muscle strength and hypertrophy. The self-selection or prescription of intensity is critical to optimal 
1 resistance training. Our data demonstrate that unsupervised trainees select loads that are

2 lower than those selected by trained subjects who train under the guidance of a personal

3 trainer. Therefore, supervised resistance training by a personal trainer appears to be

4 advantageous, when examining load selection and strength improvements. Load selection

5 should match training goals and strength training may require heavier loads than the

6 percentages observed in our sample of the population.

\section{ACKNOWLEDGEMENTS}

\section{REFERENCES}

1. Baker, D. Science and practice of coaching a strength training program for novice and intermediate-level athletes. Strength Cond J. 23: 61-68, 2001.

2. Coutts, AJ, Murphy, AJ, and Dascombe, BJ. Effect of direct supervision of a strength coach on measures of muscular strength and power in young rugby league players. $J$ Strength Cond Res, 18, 316-323, 2004.

3. Focht, BC. Perceived exertion and training load during self-selected and imposedintensity resistance exercise in untrained women. J. Strength Cond. Res. 21: 183-187, 2007.

4. Gentil, P, and Bottaro, M. Influence of supervision ratio on muscle adaptations to resistance training in nontrained subjects. J Strength Cond Res. 24: 639-643, 2010. doi: 10.1519/JSC.0b013e3181ad3373

5. Glass, SC, and Stanton, DR. Self-selected resistance training intensity in novice weightlifters. J Strength Cond Res. 18: 324-327, 2004. 
6. Gram, B, Andersen, C, Zebis, MK, Bredahl, T, Pedersen, MT, Mortensen, OS, and Sjøgaard, G. Effect of training supervision on effectiveness of strength training for reducing neck/shoulder pain and headache in office workers: cluster randomized controlled trial. BioMed Res Int, 2014:693013. doi: 10.1155/2014/693013.

7. International Health, Racquet \& Sportsclub Association [IHRSA]. IHRSA Health Club Consumer Report: 2012 Health Club Activity, Usage, Trends \& Analysis. Boston, MA: IHRSA, pp. 3-20, 2012.

8. Mazzetti, SA, Kraemer, WJ, Volek, JS, et al. The influence of direct supervision of resistance training on strength performance. Med Sci Sports Exerc. 32: 1175-1184, 2000.

9. Ratamess, NA, Alvar, BA, Evetovich, TK, Housh, TJ, Kibler, WB, Kraemer, WJ, and Triplett, NT. American College of Sports Medicine Position Stand: Progression models in resistance training for healthy adults. Med Sci Sports Exerc 41: 687-708, 2009. doi: 10.1249/MSS.0b013e3181915670

10. Ratamess, NA, Faigenbaum, AD, Hoffman, JR, and Kang, J. Self-selected resistance training intensity in healthy women: The influence of a personal trainer. $J$ Strength Cond Res. 22: 103-111, 2008. doi: 10.1519/JSC.0b013e31815f29cc.

11. Robertson, RJ, Goss, FL, Rutkowski, J, Lenz, B, Dixon, C, Timmer, J, Frazee, K, Dube, J, and Andreacci, J. Concurrent validation of the OMNI perceived exertion scale for resistance exercise. Med Sci Sports Exerc. 35: 333-341, 2003.

12. Sheppard, JM and Triplett, NT. Program design for resistance training. In Haff, GG and Triplett, NT (Eds.), Essentials of Strength Training and Conditioning (4th ed.). Champaign, IL: Human Kinetics, 2016. pp. 439-470. 
13. Schoenfeld, BJ, Wilson, JM, Lowery, RP, and Krieger, JW. Muscular adaptations in low- versus high-load resistance training: A meta-analysis. Eur J Sport Sci. 16: 1-10, 2016. doi: 10.1080/17461391.2014.989922

14. Storer, TW, Dolezal, BA, Berenc, MN, Timmins, JE, and Cooper, CB. Effect of supervised, periodized exercise training vs. self-directed training on lean body mass and other fitness variables in health club members. J Strength Cond Res. 28: 19952006, 2014. doi: 10.1519/JSC.0000000000000331.

\section{Table 1}

Descriptive Characteristics of the PT and WoPT Groups

\begin{tabular}{cccc}
\hline & PT & WoPT & t test \\
& & & (p-value) \\
\hline Age (years) & $24.1 \pm 2.9$ & $23.9 \pm 2.4$ & 0.813 \\
Height $(\mathrm{cm})$ & $167.5 \pm 8.7$ & $170.3 \pm 5.5$ & 0.377 \\
Body mass $(\mathrm{kg})$ & $65.8 \pm 10.3$ & $66.7 \pm 8.8$ & 0.828 \\
BMI (kg/m $\left.{ }^{2}\right)$ & $23.3 \pm 1.7$ & $22.9 \pm 2.6$ & 0.740 \\
Body fat (\%) & $17.1 \pm 6.3$ & $15.0 \pm 5.8$ & 0.461 \\
RT frequency (days/week) & $4.1 \pm 0.9$ & $4.1 \pm 0.8$ & 0.946 \\
\hline PT - Personal Trainer; WoPT - Without Personal Trainer; BMI - body mass index; RT - & \\
resistance training & & &
\end{tabular}


1 Table 2

2 Self-Selected Resistance Exercise Loads, 1RM and 10RM data.

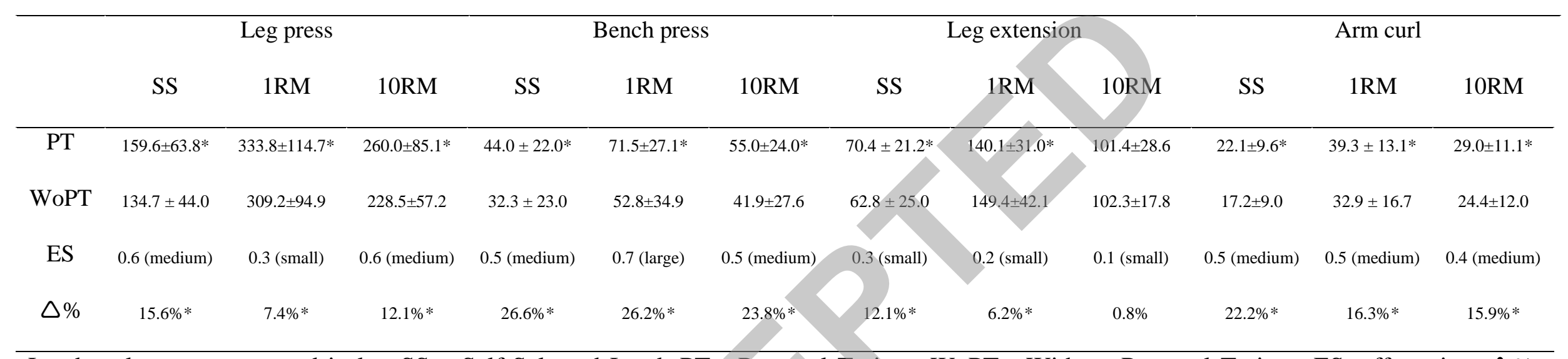

3 Loads values are presented in kg; SS - Self-Selected Load; PT - Personal Trainer; WoPT - Without Personal Trainer; ES: effect size; $\triangle \%$ :

4 percentage difference between PT and WoPT. $* P<0.05$ between groups 
1 Figure 1. Experimental design of resistance training

2

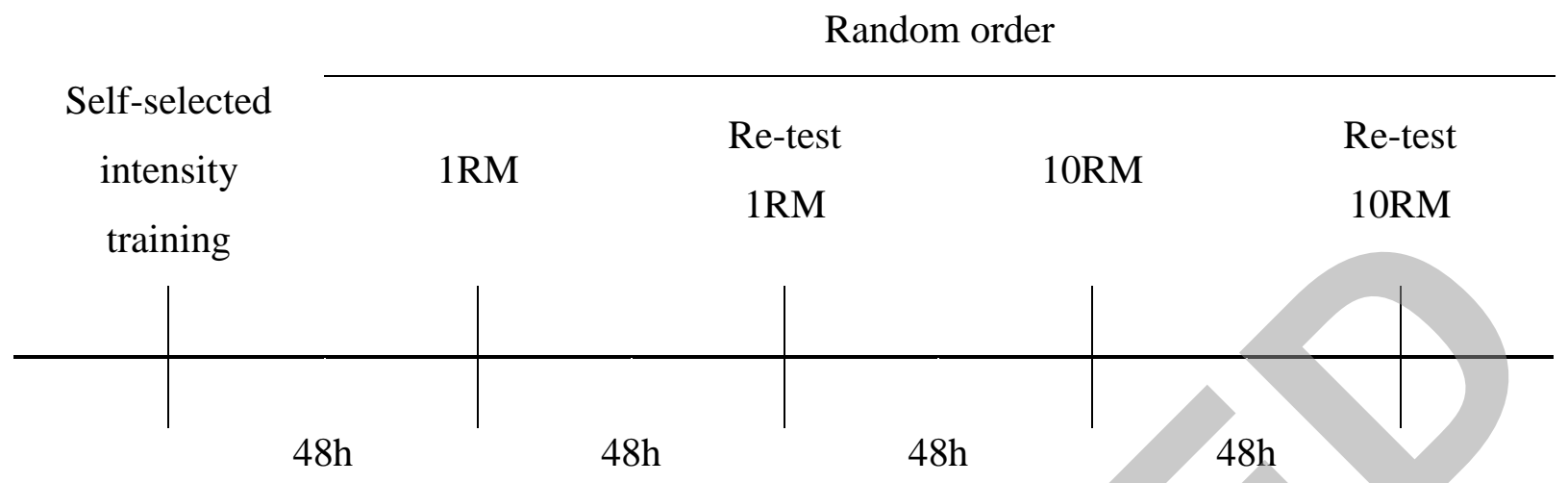

3 
1 Figure 2. Differences of (A) one-repetition maximum percentage (\%1RM) and (B) ten-

2 repetition maximum percentage $(\% 10 \mathrm{RM})$ between the personal trainer $(\mathrm{PT})$ and without personal trainer $($ WoPT) groups. $* P<0.05$ between groups. Data presented are

4 the mean \pm SD.

5 (A)

6

8

(B)

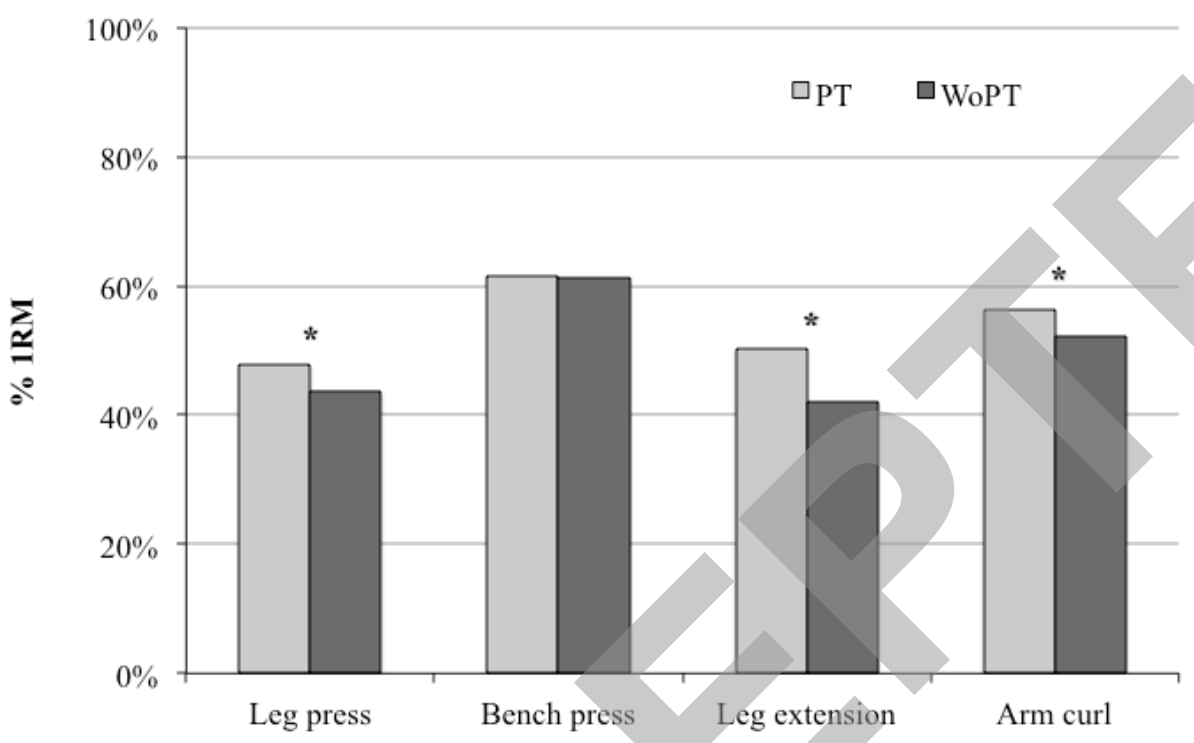

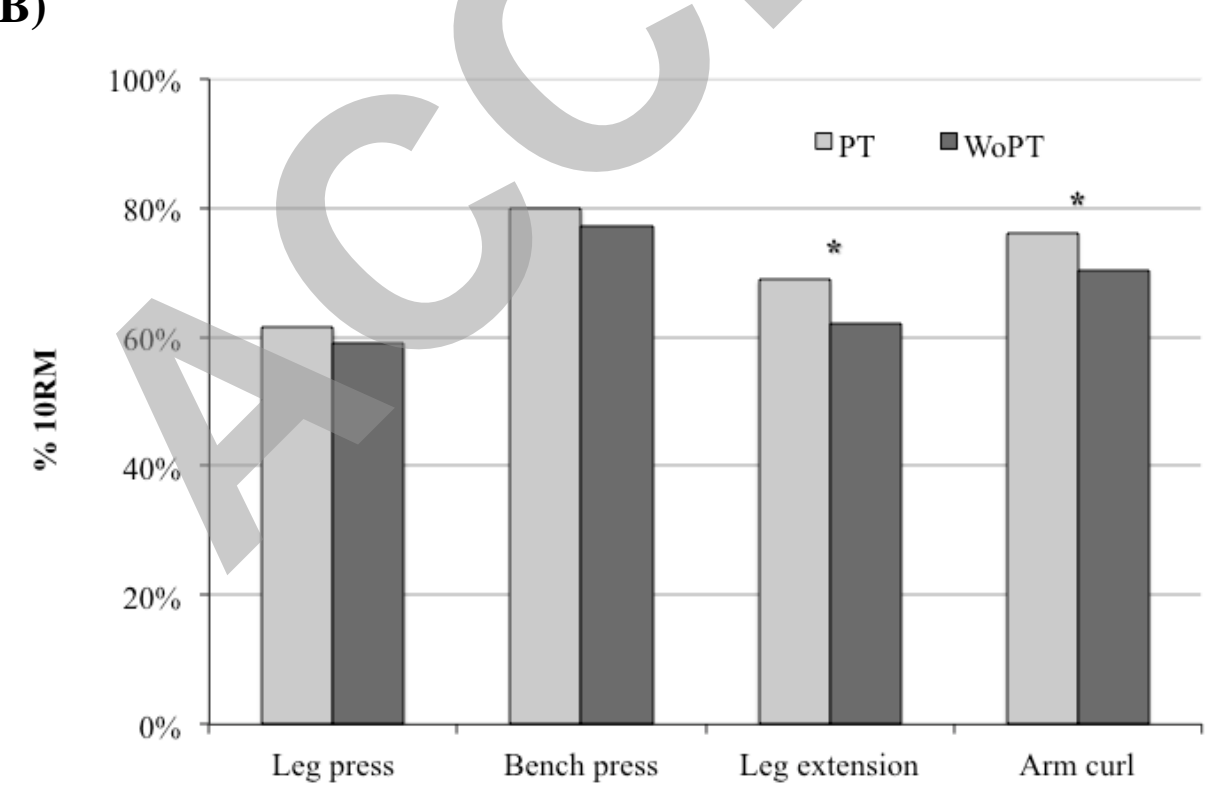

